\title{
Diversidade da macrofauna e caracterização do solo que compõe a área de Nim (Azadirachta Indica) da Fazenda Escola de Igarapé Açu no Nordeste Paraense
}

A comunidade de organismos do solo, inclui espécies visíveis a olho nu (>2,0 mm), é representada por mais de 20 grupos taxonômicos. O solo é um ambiente heterogêneo e dinâmico, na qual se constitui uma enorme variedade de organismos e alta biodiversidade e abundância de seres vivos. Esse trabalho tem como objetivo analisar a diversidade e caracterizar o solo de uma área de Nim (Azadirachta indica), localizada Igarapé-açu, fazenda escola da UFRA, por meio de índices de diversidade para a macrofauna edáfica e a carta de Munsell para identificação da cor do solo. 0 trabalho foi conduzido durante 6 meses no período de março a setembro de 2018, contendo 4 visitas ao longo da pesquisa. Com isso foram realizadas 3 coletas para macrofauna edáfica e solo. Foram utilizados os índices de Shannon-Wiener, Simpson e Pielou, para avaliar a diversidade da macrofauna, e no solo, foram feitas coletas por blocos, onde cada bloco realizou-se 4 repetições com profundidades de $0 \mathrm{~cm}-20 \mathrm{~cm}, 20 \mathrm{~cm}-40 \mathrm{~cm}, 40 \mathrm{~cm}-60 \mathrm{~cm}$ e $60 \mathrm{~cm}-80 \mathrm{~cm}$, em um total de 64 amostras. Tanto a macrofauna édafica como o solo, mostraram uma associação fundamental para o equilíbrio de um sistema onde abriga uma variedade considerável de vida. Conforme os índices de diversidade, pode-se perceber que existem grupos taxonômicos que dominante determinado habitat em prol da condição e o período que se encontram, indicando maior atividade na coleta 3 , fato atrelado ao início do período seco onde as folhas de Nim começam a cair. O solo da região foi caracterizado como latossolos com características argilo-arenosa. Foi possível, constatar que a macrofauna edáfica, associada com o meio solo como um todo, são fundamentais para o equilíbrio de um sistema que habita uma variedade inimaginável de vida. Sendo os representantes dos Diplopodas a espécie mais presente na área estudada.

Palavras-chave: Comunidade; Índices; Identificação.

\section{Diversity of macrofauna and characterization of the soil that composes the Nim area (Azadirachta Indica) of the Igarapé Açu School Farm in the Northeast of Pará}

\begin{abstract}
The community of soil organisms, including species visible to the naked eye $(>2.0 \mathrm{~mm})$, is represented by more than 20 taxonomic groups. The soil is a heterogeneous and dynamic environment, in which there is a huge variety of organisms and high biodiversity and abundance of living beings. This work aims to analyze the diversity and characterize the soil of an are of Nim (Azadirachta indica), located Igarapé-açu, UFRA school farm, through diversity indices for the edaphic macrofauna and the Munsell letter for identification of the soil color. The work was conducted for 6 months from March to September 2018, containing 4 visits throughout the survey. With this, 3 collections were made for edaphic macrofauna and soil. The ShannonWiener, Simpson and Pielou indices were used to assess the diversity of the macrofauna, and collections were made in blocks in the soil, where each block was performed 4 repetitions with depths of $0 \mathrm{~cm}-20 \mathrm{~cm}, 20 \mathrm{~cm}-40 \mathrm{~cm}, 40 \mathrm{~cm}-60 \mathrm{~cm}$ and $60 \mathrm{~cm}-80 \mathrm{~cm}$, for a total of 64 samples. Both the ephoric macrofauna and the soil, showed a fundamental association for the balance of a system where it houses a considerable variety of life. According to the diversity indexes, it can be seen that there are taxonomic groups that dominate a specific habitat in favor of the condition and the period they are in, indicating greater activity in collection 3 , a fact linked to the beginning of the dry period, where the neem leaves begin falling. The region's soil was characterized as oxisols with sandy-clay characteristics. It was possible to verify that the edaphic macrofauna, associated with the soil environment as a whole, are fundamental for the balance of a system that inhabits an unimaginable variety of life. The representatives of Diplopodas are the most present species in the studied area.
\end{abstract}

Keywords: Community; Indexes; Identification.

Topic: Organização da Biodiversidade

Reviewed anonymously in the process of blind peer.
Received: $05 / 12 / 2020$

Approved: 20/02/2021
Luan Daniel Silva Ferreira (iD

Universidade Federal do Pará, Brasil

http://lattes.cnpq.br/0794526919642626

http://orcid.org/0000-0001-9187-6988

luan.ferreirabio@gmail.com

Diocléa Almeida Seabra Silva

Universidade Federal Rural da Amazônia, Brasil

http://lattes.cnpq.br/8554224619653187

http://orcid.org/0000-0002-7102-7580

diocleaseabra85@gmail.com

Raimundo Rafael de Sousa Soares (iD)

Universidade Federal Rural da Amazônia, Brasil

http://lattes.cnpq.br/6412389435939929

http://orcid.org/0000-0002-7445-4000

rafa.biosousa@gmail.com

6

DOI: 10.6008/CBPC2318-2881.2021.001.0003
Alden Rodrigues Damasceno (D)

Universidade Federal Rural da Amazônia, Brasil

http://lattes.cnpq.br/2892259423143239

http://orcid.org/0000-0003-0591-9967

aldenrodrigues12@gmail.com
Referencing this:

FERREIRA, L. D. S.; SILVA, D. A. S.; SOARES, R. R. S.; DAMASCENO, A. R. Diversidade da macrofauna e caracterização do solo que compõe a área de Nim (Azadirachta Indica) da Fazenda Escola de Igarapé Açu no Nordeste Paraense. Nature and Conservation, v.14, n.1, p.24-31, 2021. DOI: http://doi.org/10.6008/CBPC2318-2881.2021.001.0003 


\section{INTRODUÇÃO}

O conhecimento da macrofauna edáfica e do solo, é de fundamental importância para que se possa caracterizar e avaliar os efeitos que os diferentes sistemas de cultivo e ocupação do solo pode ocasionar no ecossistema solo como um todo. Dentre os quais podemos citar o Nim (Azadirachta indica), uma espécie de origem asiática, adaptada à ambientes quentes, até mesmo em regiões semiáridas, se desenvolvendo rapidamente, atingindo de 15 a 20 metros de altura (MOURA et al., 2006). De acordo com Silva et al. (2001), o Nim tem servido para inúmeras utilidades dentre elas como matéria prima para a indústria de cosméticos e sendo considerado um inseticida natural, além de apresentar potencial para uso medicinal.

A comunidade de organismos do solo, inclui espécies visíveis a olho nu (>2,0 mm), é representada por mais de 20 grupos taxonômicos. Conforme Decaëns et al. (2008), a fauna do solo compreende uma diversidade extraordinária de tamanhos de corpos, formas de vida e comportamentos, com representantes de praticamente todos os principais táxons e grupos tróficos que formam o reino animal.

Conforme Ferreira (2015), o processo de manejo do solo, poderá modificar as estruturas de suas propriedades, alterando assim a composição e abundância da macrofauna edáfica. A macrofauna edáfica desempenha importante papel no ecossistema em se tratando do seu funcionamento, uma vez que estão inseridos em todos os níveis tróficos, do ambiente solo, sendo assim fundamentais na produção primaria, sejam afetando de maneira direta ou indireta (SILVA et al., 2007).

O solo é um ambiente heterogêneo e dinâmico, na qual se constitui uma enorme variedade de organismos e alta biodiversidade e abundância de seres vivos. Na qual a diversidade de seres do solo se insere nos processos físicos e químicos deste meio, desta maneira sofre mudanças com os processos de manejo, bem como aos diferentes tipos de uso do solo (CARDOSO et al., 1992).

Portanto, o presente trabalho teve por objetivo avaliar a diversidade da macrofauna edáfica em uma área de Nim (Azadirachta indica) na Fazenda Escola de Igarapé Açu, bem como avaliar os efeitos ocasionados em virtude do sistema de ocupação do solo.

\section{MATERIAIS E MÉTODOS}

A pesquisa foi realizada na Fazenda Escola de Igarapé-Açu (FEIGA), pertencente à Universidade Federal Rural da Amazônia (UFRA), que está localizada no município de Igarapé-Açu, nordeste do estado do Pará, possuindo coordenadas 0107'33" S e 4737'27" W, conforme a figura 1.

O solo é classificado como Latossolo Amarelo e textura média, distrófico e bem drenado. O clima local é classificado de acordo com Koppen como Am, possuindo uma precipitação média anual de 2000 a $3000 \mathrm{~mm}$, sendo assim do tipo quente e úmido, chuvoso. Caracterizada por possuir um período chuvoso (janeiro a julho), e estação seca entre setembro e dezembro, possuindo uma variação média anual de $25^{\circ} \mathrm{C}$ a $27{ }^{\circ} \mathrm{C}$ e cerca de $80 \%$ a $90 \%$ de umidade relativa (KATO et al., 2011; SOUZA et al., 2011; PEREIRA et al., 2012). 


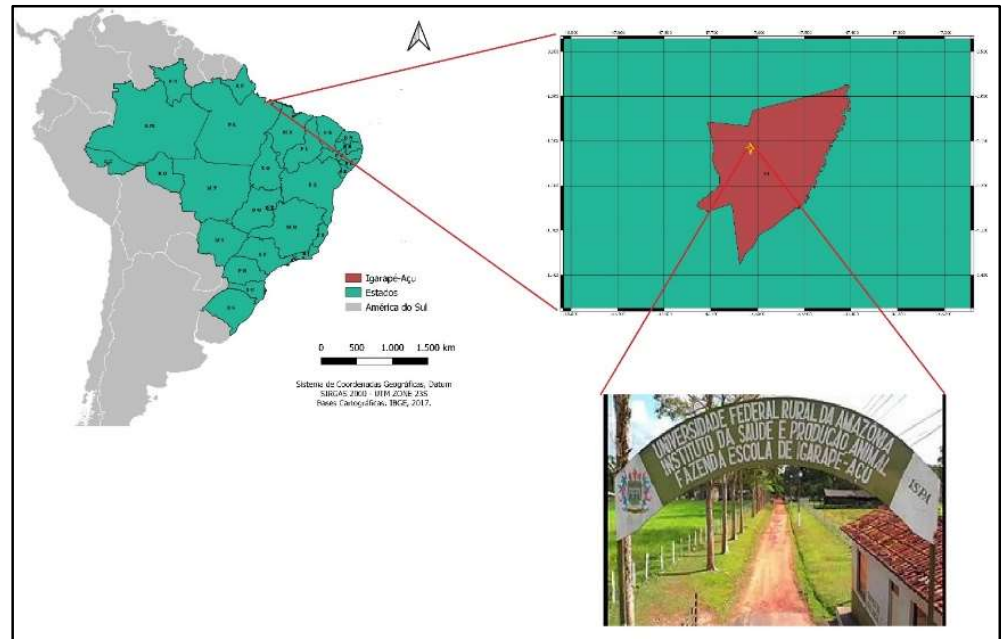

Figura 1: Mapa da localização da escola fazenda da UFRA.

O trabalho foi conduzido durante 6 meses no período de março a setembro de 2018, contendo 4 visitas ao longo da pesquisa, nos meses de março, abril, junho e agosto. À primeira vista, consistiu apenas na identificação da área a ser conduzido o experimento. No segundo momento (abril/2018), já com a identificação e escolha da área, foram definidos o número de blocos que seriam trabalhados na área de estudo. Perfazendo um total de 4 blocos, sendo que cada bloco possuindo comprimento $5 \times 5$ metros $\left(25 \mathrm{~m}^{2}\right)$. Para delimitar cada área dos blocos $5 \times 5$ metros, utilizou-se de uma trena de 50 metros para facilitar o processo de demarcação do local escolhido.

Cada bloco possuía quatro parcelas, essas parcelas consistiam em repetições de cada bloco. As parcelas eram de $1 \times 1$ metros $\left(1 \mathrm{~m}^{2}\right)$. Em cada parcela foram realizadas tradagens, dos horizontes do solo para coleta de amostragem de solo necessárias para classificação, assim como também coleta da macrofauna edáfica. Nas duas últimas visitas subsequentes, (junho/2018) e (setembro/2018), foram realizadas apenas coletas de amostragens edáficas.

Para coletar as amostras de solo de cada repetição por bloco, utilizou-se de um trado Holandês. O material foi retirado com o auxílio do trado, obedecendo a uma sequência de profundidade de 0 20, 20 40, 40 60 e 60 80 cm (NANNI et al., 2015). O procedimento foi realizado em todas as parcelas de cada bloco, desta maneira obtendo 16 amostras de solo de cada bloco e, um total de 64 amostras dos 4 blocos ao longo do estudo. As tradagens consistiram da seguinte maneira: bloco $1(5 \times 5 \mathrm{~m})$, parcela 1 (R1), Parcela 2 (R2), Parcela 3 (R3), Parcela 4 (R4). E assim prosseguiu para os demais blocos.

O material coletado de solo em campo, foram armazenados em sacos plásticos, sendo que todas as amostras foram previamente identificadas com canetas permanentes, por repetição e blocos retirados, assim como colocadas em copos descartáveis, (Figura 1) para facilitar as análises posteriores em laboratório e que não ocorresse possíveis trocas de amostra. Os exemplares de macrofauna coletadas, em cada repetição dos blocos foram armazenadas em copos descartáveis a álcool 70\%, para conservação das amostras invertebrada (NAZARO et al., 2010).

Nas análises em laboratório, os exemplares de macrofauna invertebrada, foram devidamente armazenados em copos descartáveis com álcool 70\% para fim de conservação das amostras, todos os copos, 
estavam identificados com etiquetas, com sua descrição de qual bloco, assim como qual repetição pertencia. Todas as amostras foram classificadas a nível de ordem e família, conforme a chave de identificação de espécies e do manual de entomologia agrícola. Após realizada a etapa de classificação, realizou-se o procedimento medição de cada espécie coletada, isso foi possível com o auxílio de um paquímetro digital modelo ZASS Precision 6", (Figura 2).

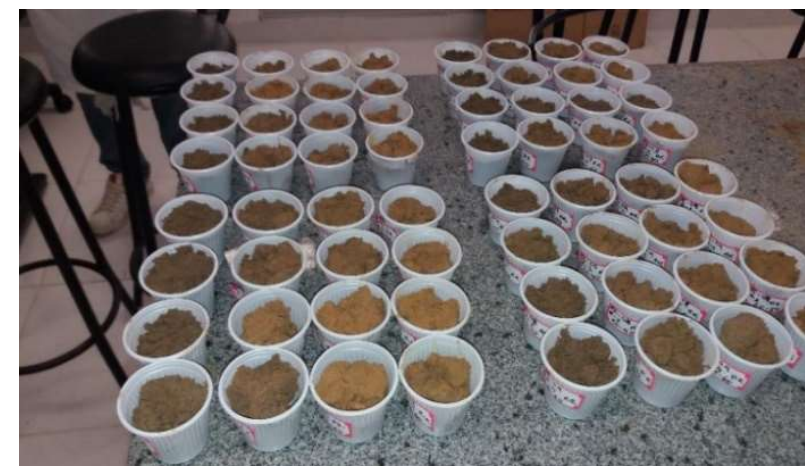

Figura 1: Amostras de solo dos 4 blocos analisados.

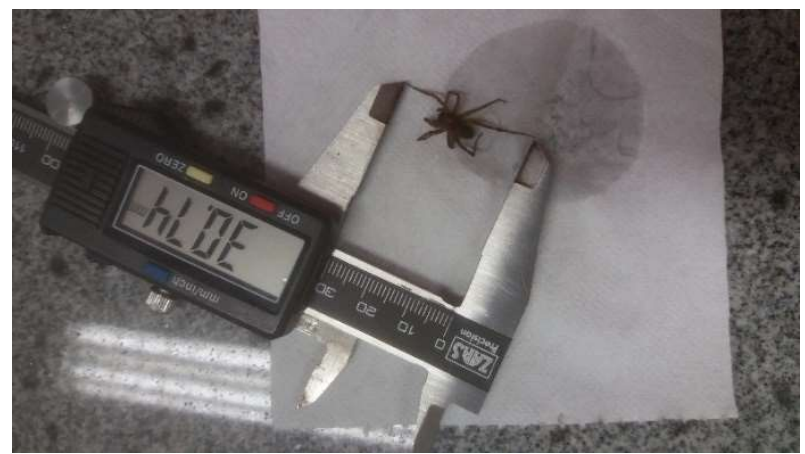

Figura 2: Modelo de paquímetro utilizado para medir a macrofauna.

A partir dos resultados obtidos, calcularam-se a riqueza da macrofauna (riqueza numérica dos grupos identificados) e os índices de diversidade de Shannon-Wiener $\left(\mathrm{H}^{\prime}\right)$, de equitabilidade de Pielou. $\mathrm{O}$ índice de Shannon foi estimado pela fórmula: $\mathrm{H}^{\prime}=-\sum(\mathrm{Xi} / \mathrm{Xo}) \times \log (\mathrm{Xi} / \mathrm{Xo})$, onde: $\mathrm{Xi} / \mathrm{Xo}$, representa a proporção do grupo " $\mathrm{i}$ " no total das amostras. $O$ índice de dominância de Simpson foi estimado a partir da fórmula: $\mathrm{D}=\Sigma(\mathrm{Xi} / \mathrm{Xo})^{2}$. Este índice reflete a probabilidade de dois indivíduos escolhidos ao acaso na comunidade pertencerem à mesma espécie e varia de 0 a 1 e, quanto mais alto for, maior a probabilidade de os indivíduos serem da mesma espécie, ou seja, maior a dominância e menor a diversidade. $O$ índice de Pielou foi estimado a partir da fórmula: $\mathrm{U}=\mathrm{H}^{\prime} / \log \mathrm{S}$, onde $\mathrm{S}$ é o número total de ordens identificadas em cada sistema estudado.

Para a análise do solo as amostras coletas em campo, de cada bloco foram submetidas a identificação por meio do guia de textura ao tato elaborado por Rowell 1994, que consiste em seguir alguns passos que é pegar uma pequena amostra do solo, levar a mão molha-lo para facilitar o manuseio, e seguir a sequência do guia, dentre eles verificar se o solo forma ou não bolas coesas, cilindros finas e filamentos, se ele molda em "U", forma "anel" e se forma é com "fendilha" ou não, para que se possa chegar a uma definição de qual solo estamos analisando. Cada amostra analisada segundo o guia de textura fora identificada por etiquetas a qual bloco e repetição pertencia, bem como de qual perfil de solo e, armazenado em um copo descartável, para que fosse posteriormente definido segundo a cor de cada solo, seguindo a Carta de Munsell (FERNANDES et al., 2004; NANNI et al., 2015).

\section{RESULTADOS E DISCUSSÃO}

De acordo com os resultados obtidos nas análises do tamanho corporal das amostras de macrofauna edáfica. Na primeira coleta obteve-se uma variação de $10,66 \mathrm{~cm}$ a 48,81 cm de comprimento corpóreo, totalizando 19 indivíduos. Já na segunda amostragem a média de comprimento corporal variou de 4,66 cm a $39.10 \mathrm{~cm}$, com representantes do grupo dos Coleóptera e Diplopoda, respectivamente. A última análise dos 
exemplares da coleta da pesquisa, constatou-se uma variação de 5,33 cm a 33,22 cm de extensão corpóreo.

Após realizada a classificação e contagem de todas as amostras de macrofauna ao longo da pesquisa. Constatou-se uma ligeira predominância dos representantes dos Myriapoda/Diplopoda com 97 exemplares no total, durante o período das coletas. Isso pode ser explicado em função da grande humidade na área estudada, visto que as duas primeiras visitas coincidiram com o período chuvoso da região, o que conferiu um ambiente propicio para a espécie. Fato este não identificado na terceira visita, uma vez que coincidiu com período seco. Logo em seguida, os Arachnida perfizeram exatamente 29 indivíduos. Os menos encontrados foram Lepidóptera e Isopoda, com apenas 1 representante cada. Conforme contagem, o total amostral da coleta 1, foi de 48 exemplares, a segunda com 83 e a última com 37, totalizando 168 espécies coletadas (Tabela 1).

Tabela 1: Principais grupos taxonômicos coletados durante a pesquisa.

\begin{tabular}{lllll}
\hline Grupos & Coleta 1 & Coleta 2 & Coleta 3 & No total \\
\hline Arachnida & 6 & 8 & 15 & 29 \\
Blattodea & 0 & 4 & 4 & 8 \\
Coleoptera & 0 & 2 & 1 & 3 \\
Diplopoda & 35 & 59 & 3 & 97 \\
Gastropoda & 1 & 4 & 3 & 8 \\
Hymenoptera & 3 & 3 & 4 & 10 \\
Hemiptera & 0 & 1 & 7 & 8 \\
Isopoda & 0 & 1 & 0 & 1 \\
Lepidóptera & 0 & 1 & 0 & 1 \\
Orthoptera & 3 & 0 & 0 & 3 \\
\hline
\end{tabular}

As maiores riquezas de grupos foram detectadas na coleta 2 com 83 indivíduos, e a menor foi a coleta 3 com 37. O número máximo de grupos encontradas nas 3 foi 10 , com número mínimo de 5 na primeira coleta, sendo considerado baixo em relação a outros trabalhos realizados na região Norte brasileira (MATHIEU et al., 2005; BARROS et al., 2008).

O índice de diversidade de Shannon $\left(H^{\prime}\right)$ é um dos mais comumente usados e mostra-se, extremamente, apropriado para o uso em ecologia do solo, uma vez que atribui maiores valores às espécies raras presentes na comunidade (TOLEDO, 2003). $O$ índice $H^{\prime}$ foi alto nos na coleta 3 (período seco), alcançado valores iguais nas coletas 1 e 2 durante o período chuvoso, indicando maior atividade da macrofauna no período seco (Tabela 2). Esse fato ocorre devido a arvore Nim (Azadirachta indica) perder suas folhas no período seco, ao mesmo tempo que os brotos foliares surgem (NEVES et al., 2009).

Tabela 2: Índices de diversidade dos grupos da macrofauna do solo de Shannon-Wiener, de Simpson e de Pielou, referentes as 3 coletas realizadas.

\begin{tabular}{lllll}
\hline Coleta & Shannon & Simpson & Pielou & riqueza \\
\hline 1 & 0,39 & 0,44 & 0,23 & 48 \\
2 & 0,39 & 0,47 & 0,2 & 83 \\
3 & 0,72 & 0,76 & 0,46 & 37 \\
\hline
\end{tabular}

O maior valor do índice de Simpson (D) foi na coleta 3, durante a estação seca, sendo o mais alto de todas as coletas. Desse modo, a equitabilidade dos grupos foi baixa nesta coleta e época, determinando a dominância do grupo Arachnida em relação aos demais grupos encontrados.

O índice de Pielou mostrou-se baixo na primeira e segunda coleta. Isto indica a dominância de um 
Diversidade da macrofauna e caracterização do solo que compõe a área de Nim (Azadirachta Indica) da Fazenda Escola de lgarapé Açu no

grupo sobre os outros, sendo o grupo Diplopoda o mais dominante. Muitas vezes esse fato pode estar relacionado com as épocas de coleta das amostras de solo para análise da fauna, o qual tem influência direta na densidade, riqueza, índices de Shannon e de Pielou (MOÇO et al., 2005). Foi observado que quando o índice de Pielou diminuiu, para uma dada riqueza, a diversidade aumenta com o aumento da equitabilidade e, da mesma forma, para uma dada equitabilidade, muitas vezes, a diversidade aumenta proporcionalmente à riqueza (BEGON et al., 1986).

De acordo com Lee (1994), os organismos da macrofauna edáfica apresentam comportamento sazonal ou são ativos apenas em determinados períodos do ano. Podem também apresentar caráter oportunista, explorando condições favoráveis o solo para aumentarem rapidamente suas populações, as quais podem, logo em seguida, serem diminuídas novamente (LEE, 1994). Para Assad (1997), a sazonalidade pluviométrica também afeta essas populações, visto que a água é o principal fator limitante da sua atividade.

A textura do solo é uma caraterística extremamente relevante, sendo fundamental seu conhecimento, para os processos de classificação e caracterização. De acordo com Fujiyama et al. (2010) a textura é uma das características mais importantes que o solo possui, sendo as frações de areia, silte e argila, os fatores determinantes para se definir seu aspecto textural. Nesse sentido conforme analises do guia de textura ao tato, obteve-se uma variação textural de Areia Franca (AF) nas profundidades de $0 \mathrm{~cm}-20 \mathrm{~cm}$, e Argila (A) de $60 \mathrm{~cm}-80 \mathrm{~cm}$.

As cores do solo é um dos atributos, assim como da textura fundamentais, seja para levantamento, classificação ou caracterizar esse meio. Conforme Botelho et al. (2006), A cor é uma das mais importantes características do solo, sendo considerada na sua identificação e na sua descrição no campo, além de ser um atributo diferencial para muitas classes de solos nos sistemas de classificação. De acordo com Campos (2001) a coloração apresentada pelos solos constitui uma das maneiras mais primitivas de identificá-los. O mesmo autor menciona que as cores apresentadas pelos solos, estar intimamente ligado a presença de óxidos de ferro, sobretudo os componentes mineralógicos hematita e goethita, isso tem despertado o interesse de inúmeros pesquisadores com intuito de determinar a cor dos solos de forma mais exata, estabelecendo uma relação quantitativa entre a cor e seus constituintes mineralógicos.

Nesse contexto, seguindo as colorações presentes na carta de Munsell, obteve-se diferentes tipos de padrões de cores a depender da profundidade da amostra de solo coletada, possuindo uma variação de 2.5 Y 4 / / 3 a 2.5 Y 6 / / 6, nos blocos 2, 3 e 4 nas profundidades de $0 \mathrm{~cm}-20 \mathrm{~cm}$ a $0 \mathrm{~cm}-80 \mathrm{~cm}$, e 2.5 Y 5 / / 3 a 2.5 Y 5 / / 3, no bloco 1, nas profundidades de $20 \mathrm{~cm}-40 \mathrm{~cm}, 40 \mathrm{~cm}-60 \mathrm{~cm}$ e $60 \mathrm{~cm}-80 \mathrm{~cm}$

\section{CONCLUSÕES}

Foi possível, constatar que a macrofauna edáfica, associada com o meio solo como um todo, são fundamentais para o equilíbrio de um sistema que habita uma variedade inimaginável de vida. Sendo os representantes dos Diplopodas a espécie mais presente na área estudada. Isso pode ter uma ligação direta com o período de coleta, chuvoso, que conferiu um ambiente essencial para desenvolvimento da espécie. 0 
solo da região é do tipo latossolos se destacando com característica argilo-arenosa. $\mathrm{O}$ índice de diversidade de Shannon indica que a macrofauna do solo tem maior atividade na coleta 3 , início do período seco devido ao maior aporte de serapilheira.

Portanto os estudos ao longo desses 6 meses foram de extremo valor, sendo fundamental que sejam desenvolvidos mais trabalhos e pesquisas mais aprofundados naquela área. Com técnicas e análises mais detalhadas, sobretudo da relação que a macrofauna exerce na característica do solo, assim como uso de ademais análises nas amostras de solo, a exemplo das químicas.

\section{REFERÊNCIAS}

ASSAD, M. L. L.. Fauna do solo. In: VARGAS, M. A. T.; HUNGRIA, M.. Biologia dos solos dos Cerrados. Planaltina: EMBRAPA-CPAC, 1997. p.363-443.

BEGON, M. J. L.; HARPER, J.L.; TOWNSEND, C. R.. Ecology: Individuals, populations and communities. 3 ed. Oxford, Blackwell Science, 1986.

BOTELHO, M. R.; DALMOLIN, R. S. D.; PEDRON, F. A.; AZEVEDO, A. C.; RODRIGUES, R. B.; MIGUEL, P.. Medida da cor em solos do Rio Grande do Sul com a carta de Munsell e por colorimetria. Ciência Rural, Santa Maria, v.36, n.4, p.1179-1185, 2006.

CARDOSO, E. J. B. N.. Microbiologia do solo. 2 ed. São Paulo, 1992.

\section{CAMPOS, R. C.. Determinação da cor do solo e sua} utilização na predição dos teores de hematita. Dissertação (Mestrado em Agronomia) - Universidade de São Paulo, Piracicaba, 2001.

DECAËNS, T.; LAVELLE, P.; JIMÉNEZ, J. J.. Priorities for conservation of soil animals. CAB Reviews: Perspectives in Agriculture, Veterinary Science, Nutrition and Natural Resources, 2008.

FERREIRA, S. A.. Avaliação visual da estrutura e macrofauna edáfica em sistemas de uso do solo no sub-trópico Brasileiro. Dissertação 9Mestrado em ciências do solo) Universidade Federal do Paraná, Curitiba, 2015.

FERNANDES, R. B. A.; BARRÓN, V.; TORRENT, J.; FONTES, M. P. F.. Quantificação de óxidos de ferro de latossolos brasileiros por espectroscopia de refletância difusa. R. Bras. Ci. Solo, v.28, p.245-257, 2004.

FUJIYAMA, B. S.; TAVARES, M. M.. Caracterização qualitativa por difração de raios- $x$ de amostras de solos: condições mineralógicas ao benefício do ambiente. In: CONGRESSO ANUAL DA ABM, 65. Anais. Rio de Janeiro, 2010.

KATO, O. R.; SHIMIZU, M. K.; BORGES, A. C. C. R.. Produção de raízes de mandioca em sistemas agroflorestais sequenciais submetidos a diferentes manejos de adubações verde e fertilizantes no nordeste paraense. Embrapa Amazônia Oriental, 2011.

LEE, K. E.. The biodiversity of soil organisms. Applied Soil Ecology, v.1, p.251-254, 1994.
MATHIEU, J.; ROSSI, J. P.; MORA, P.; LAVELLE, P.; MARTINS, P. F. D.; ROULAND, C.; GRIMALDI, M.. Recovery of soil macrofauna communities after forest clearance in Eastern Amazonia, Brazil. Conservation Biology, v.19, p.1598-1605, 2005.

MOURA, M.; SOARES, T.. Uso do Nim uma árvore de múltiplas utilidades. Ouricuri: Caatinga Centro de Assessoria e Apoio aos Trabalhadores e Instituições Não Governamentais Alternativas, 2006.

MOÇO, M. K. S.; GAMA-RODRIGUES, E. F.; GAMARODRIGUES, A. C. G.; CORREIA, M. E. F.. Caracterização da fauna edáfica em diferentes coberturas vegetais na região norte Fluminense. Revista Brasileira Ciência do Solo, v.29, p.555-564, 2005.

NANNI, M. F.; DEMATTÊ, J. A. M.; JUNIOR, C. A. S.; ROMAGNONI, F.; SILVA, A. A.; CEZAR, E.; GASPAROTO, A. C.; CAMPOS, R. M.; SACIOTO, M.; CHICALI, M. L.; OLIVEIRA, B. R.. Comparação entre mapas de solos obtidos por métodos tradicionais e por sensores remotos orbital e terrestre. In: SIMPÓSIO BRASILEIRO DE SENSORIAMENTO REMOTO SBSR, 17. Anais. João Pessoa: INPE, 2015.

NAZARO, C. M.; MORIYA, M. R.; ALBUQUERQUE, L. B.. Macrofauna edáfica bioindicadora de qualidade de solo em sistemas orgânicos de produção de café sombreado e a pleno sol. In: ENIC. Anais. 2010.

NEVES, E. J. M.; CARPANEZZI, A. A.. Prospeç̧ão do cultivo Nim (Azadirachta indica) no Brasil. Colompo: Embrapa Florestas, 2009.

PEREIRA, M. E. M.; VASCONCELOS, S. S.; SILVA, A. K. L.; SOUZA, C. M. A.. Estoque de carbono do solo em sistema agroflorestal sequencial no nordeste Paraense. In: ENCONTRO AMAZÔNICO DE AGRÁRIAS,4. Anais. 2012.

SILVA, R. F.; TOMAZI, M.; PEZARICO, C. R.; AQUINO, A. M.; MERCANTE, F. M.. Macrofauna invertebrada edáfica em cultivo de mandioca sob sistemas de cobertura do solo. Pesq. Agropec. Bras., Brasília, v.42, n.6, p.865-871, 2007.

SILVA, H. D.; FERREIRA, C. A.; BELLOTE, A. F. J.; SANTANA, D. L. Q.; NAGEL, R.. Comportamento de procedências de Nim (Azadirachta indica A. Juss) em Jaraguá do Sul, SC. Bol. Pesq. Fl., Colombo, n.43, p.141-143, 2001. 
Diversidade da macrofauna e caracterização do solo que compõe a área de Nim (Azadirachta Indica) da Fazenda Escola de Igarapé Açu no Nordeste Paraense

FERREIRA, L. D. S.; SILVA, D. A. S.; SOARES, R. R. S.; DAMASCENO, A. R.

SOUZA, C. M. A.; FREITAS, F. S.; CARVALHO, C. J. R.; VASCONCELOS, S. S.; KATO, O. R.. Atributos físicos do solo em sistemas agroflorestais sequenciais no município de Igarapé Açú, Pará. In: CONGRESSO BRASILEIRO DE SISTEMAS AGROFLORESTAIS, 8. Anais. Belém: Embrapa Amazônia Oriental, 2011.
TOLEDO, L. O.. Aporte de serrapilheira, fauna edáfica e taxa de decomposição em áreas de floresta secundária no município de Pinheiral, RJ. Dissertação (Mestrado em Ciências Ambientais e Florestais) - Universidade Federal Rural do Rio de Janeiro, Seropédica, 2003.

A CBPC - Companhia Brasileira de Produção Científica (CNPJ: 11.221.422/0001-03) detém os direitos materiais desta publicação. Os direitos referem-se à publicação do trabalho em qualquer parte do mundo, incluindo os direitos às renovações, expansões e disseminações da contribuição, bem como outros direitos subsidiários. Todos os trabalhos publicados eletronicamente poderão posteriormente ser publicados em coletâneas impressas sob coordenação da Sustenere Publishing, da Companhia Brasileira de Produção Científica e seus parceiros autorizados. Os (as) autores (as) preservam os direitos autorais, mas não têm permissão para a publicação da contribuição em outro meio, impresso ou digital, em português ou em tradução. 